\title{
A Second-Order Statistical Method for Spectrum Sensing in Correlated Shadowing and Fading Environments
}

\author{
Khalid Qaraqe $\mathrm{e}^{\ddagger}$ and Serhan Yarkan ${ }^{\ddagger \ddagger}$ \\ $\ddagger$ Department of Electrical and Computer Engineering, Texas A \& M University at Qatar, \\ Texas A \& M Engineering Building, Education City, Doha, 23874, Qatar \\ E-mail: kqaraqeetamu. edu \\ $\ddagger \ddagger$ Electrical \& Computer Engineering, Texas A \& M University \\ 214 Zachry Engineering Center, College Station, TX, 77843-3128 \\ E-mail: syarkandece.tamu.edu
}

\begin{abstract}
Spectrum sensing is one of the most important tasks of cognitive radios (CRs) in future wireless systems and of user equipments (UEs) in next generation wireless networks (NGWNs). Therefore, deciding whether a specific portion of radio frequency (RF) spectrum is occupied or not is of paramount importance for all sorts of future wireless communications systems. In this study, a spectrum sensing method that employs a second-order statistical approach is proposed for detecting fast fading signals in spatially correlated shadowing environments. Analysis and performance results are presented along with the discussion related to the performance comparison of energy detection method.

Index Terms - fast fading, mobility, shadowing, spectrum sens-
\end{abstract} ing

\section{INTRODUCTION}

One of the most important resources for radio communications is the radio frequency $(\mathrm{RF})$ spectrum. With the increasing demand for wireless applications and services, various wireless devices and technologies need to utilize the same portion of the RF spectrum. For instance, Bluetooth, Wi-Fi (IEEE $802.11 \mathrm{~b} / \mathrm{g} / \mathrm{n}$ ), ZigBee (IEEE 802.15.4), cordless telephones, home microwave ovens, and baby monitoring devices operate on industrial, scientific, and medical (ISM) band (at $2.4 \mathrm{GHz}$ ). It is not difficult to predict that the number of technologies coexisting on the same portion of RF spectrum will increase as new wireless applications and services emerge.

At first glance such a dense coexistence seems to cause a scarcity in the RF spectrum. However, measurements reveal that the RF spectrum is actually underutilized rather than being scarce [1]. Recently, cognitive radio (CR) is proposed as a remedy to overcome this spectral underutilization concern [2]. Although there is no formal definition for CR, the term implies a radio that can sense, be aware of, and learn about its surrounding environment and adapt its parameters accordingly [3]. In this regard, one of the fundamental tasks of CR is to be aware of the RF spectrum by steadily sensing it in order to take advantage of available spectral opportunities for transmission.

Even though the notion "spectrum sensing" is coined for $\mathrm{CR}$, it is extremely important for next generation wireless networks (NGWNs) as well. In NGWNs, frequency reuse of one (FRO) is considered to be the prominent deployment strategy to avoid expensive network planning process. However, FRO comes at the expense of significant co-channel interference (CCI) levels especially for the user equipments (UEs) residing in the vicinity of cell borders. Significant CCI levels cause very poor signal reception due to a low carrier-to-interference ratio (C/I), reduced system capacity, more frequent handoffs, and dropped calls. Because CCI is inevitable in FRO schemes, it needs to be managed by the following strategies: interference avoidance, interference minimization, or interference cancellation. Therefore, identifying the unused radio resources, ${ }^{1}$ which can be regarded as a special case of spectrum sensing, plays a crucial role in interference management for NGWNs.

Spectrum sensing methods can coarsely be categorized as follows: (C1) matched filtering, (C2) waveform-based sensing, (C3) cyclostationary- and autocorrelation-based (or secondand higher-order statistical methods based) sensing, and (C4) energy detection. Although matched filtering provides an optimum way of spectrum sensing [4], it requires the knowledge of various characteristics of the transmitted signal such as signaling bandwidth, operating frequency, modulation type and order, pulse shape, and frame/burst format. Waveform-based sensing rests upon searching for some of the known characteristics of the transmitted signal such as pilot sequences or midambles throughout the received signal. Searching operation is carried out by specially constructed templates via patternmatching techniques [5, 6]. As in matched filtering, major drawback of the methods falling into $(\mathrm{C} 2)$ is the necessity of having the knowledge of some distinct characteristics of the transmitted signal. Cyclostationarity-based approaches strive to exploit the periodicity in the statistics of a signal such as its autocorrelation [7, 8]. Despite the fact that it is a very powerful method, cyclostationarity-based methods might be relatively more computationally complex. Similar to cyclostationaritybased methods, correlation-based approaches can also be applied to spectrum sensing problems $[9,10]$. However, spatial correlation of shadowing needs to be taken into account, since it changes the statistical characteristics of signals and affects the performance. Energy detection, in contrast to (C1)-(C3), adopts a very simplistic approach by comparing the energy of the received signal with a threshold [11, 12]. In spite of its performance shortcomings, it is evident that (C4) requires relatively less computationally complex operations compared to the methods classified in (C1)-(C3) ${ }^{2}$

In this study, an autocorrelation-based spectrum sensing method is proposed for a mobile receiver which strives to detect the absence/presence of an unknown transmitted signal in a general radio propagation environment. The method proposed takes into account second-order statistics of the propagation mechanisms such as spatially correlated shadowing and fast fading in the received signal. Considering

\footnotetext{
${ }^{1}$ For instance, such resources correspond to resource blocks (timefrequency units) in Third Generation Long Term Evolution (3GLTE) downlink.

${ }^{2} \mathrm{~A}$ very comprehensive survey regarding this topic along with some other prominent methods can be found in [13, and references therein].
} 
the fact that shadowing process changes slowly compared to the fast fading process, the method proposed separates the statistics of these processes from the received signal by employing a low-pass filter followed by logarithmic detector and investigating second-order statistics of the output. It is shown that in the absence of an unknown signal, the method proposed causes second-order characteristics of noise process to converge a constant that depends on the filtering duration. When an unknown signal is present, on the other hand, shadowing correlation never allows the second-order statistics to cross below that constant and identifies the presence of the unknown signal. Numerical results also show that the method proposed outperforms the conventional energy detector for the signals buried into noise. The rest of the paper is organized as follows: Section II expresses statement of the problem and provides signal model along with its statistical characteristics. Section III gives the details of the method proposed along with relevant analysis. Section IV presents the numerical results including performance comparison and relevant discussions. Section V summarizes the observations and concludes the paper.

\section{Statement of the Problem, Signal Model, And ITS STATISTICAL CHARACTERISTICS}

Statement of the Problem and Signal Model: Due to the ambient noise present over the transmission medium, complex baseband equivalent of the received signal can be represented as:

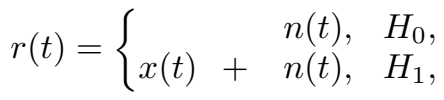

where $n(t)$ is complex additive white Gaussian noise (AWGN) with $\mathcal{C N}\left(0, \sigma_{N}^{2}\right)$ in the form of $n(t)=n_{I}(t)+j n_{Q}(t)$ as both $n_{I}(t)$ and $n_{Q}(t)$ being $\mathcal{N}\left(0, \sigma_{N}^{2} / 2\right)$ and $j=\sqrt{-1} ; x(t)$ is the complex baseband equivalent of the unknown signal; $H_{0}$ represents the hypothesis corresponding to absence of the unknown signal, whereas $H_{1}$ refers to the hypothesis corresponding to presence of it. Then, statement of the problem can be expressed as identifying the absence/presence of the unknown signal $x(t)$ by looking at the statistical characteristics of the received signal $r(t)$ in the presence of ambient noise $n(t)$.

Having said that, the unknown signal $x(t)$ can be modeled by decomposing it into the following form under the narrowband channel assumption:

$$
x(t)=m(t) s(t) a(t),
$$

where $m(t)=h(t) e^{j \theta(t)}$ represents complex fading channel process whose amplitude and phase are $h(t)$ and $\theta(t)$, respectively; $s(t)$ denotes the real-valued slow-fading process including the combined effects of both distance-dependent path loss and shadowing; and $a(t)$ is the unknown baseband signal. In addition, all three processes in (2) are assumed to be independent of each other and of the noise process $n(t)$.

Statistical Characteristics of Fast-Fading Process $[m(t)]$ : Transmitted signals arrive at the receiver through multiple rays (sometimes referred to as paths) in the wireless propagation environment. These rays are combined at the receiver antenna and form a complex fading channel process $m(t)$ representing a change in both amplitude and phase of the received signal. Complex fading channel process manifests itself by causing rapid fluctuations in the power level of the received signal with respect to very small displacements on the order of a couple of wavelengths of the transmission. Assuming that there is sufficiently large number of independent rays arriving at the receiver antenna in the absence of a specular component such as in line-of-sight (LOS) conditions, fading channel amplitude $h(t)=|m(t)|$ follows the Rayleigh distribution according to the central limit theorem. Furthermore, due to mobility, fading channel process exhibits correlation in time (or depending on the formalization, in space) [14]. As an idealized case, angleof-arrival (AoA) of the rays at an omni-directional receiver antenna can be assumed to be uniformly distributed within $[0,2 \pi)$ on a two-dimensional plane. This special case leads to the very well-known Jakes' Doppler spectrum implying a correlation in time for the complex fading channel process with $R_{h}(\tau)=J_{0}\left(2 \pi f_{D}|\tau|\right)$, where $J_{0}(\cdot)$ is the zeroth-order Bessel function of the first kind; $f_{D}$ is the maximum Doppler frequency given by $f_{c} v / c, f_{c}$ is the transmission frequency, $v$ is the mobile speed, and $c$ is the speed of transmitted waves (in RF propagation $c=3 \times 10^{8} \mathrm{~m} / \mathrm{s}$ ). Depending on the $f_{D}|\tau|$ value, one can conclude that fast-fading channel process decorrelates with itself over displacements on the order of a couple of wavelengths of the transmission [14].

Statistical Characteristics of Slow-Fading Process $[s(t)]$ : Fluctuations in the power level of the received signal is caused not only by fast-fading process but also by the transmitterreceiver separation and by the obstacles present in between. Transmitter-receiver separation causes a power loss in the received signal which is known as distance-dependent path loss and decreases monotonically with respect to the distance between transmitter and receiver. On the other hand, the impact of obstacles in the propagation paths between transmitter and receiver manifests itself as drastic fluctuations in the power level of the received signal and is called shadowing (or sometimes shadow fading). However, fluctuations in the received signal power caused by distance-dependent path loss and shadowing take place with larger displacements compared to those caused by fast-fading process. In other words, for the same speed, combined impact of path loss and shadowing exhibits a slower rate of change in the received signal power level compared to that of fast-fading process; therefore, it is referred to as "slow-fading" process. As frequently reported and verified in most of the experimental studies present in the literature, the first-order statistics of the slow-fading process can be approximated by a log-normal distribution. Therefore, the combined impact of path loss and shadowing can be captured by the following single process $[15,16]$ :

$$
s(t)=\exp \left(\frac{1}{2} \mu(t)+\frac{\sigma_{G}}{2} g(t)\right),
$$

where $\mu(t) / 2$ denotes mean, $\sigma_{G} / 2$ is the standard deviation of $\log$-normal shadowing, and $g(t)$ is a real-valued unit normal process $\mathcal{N}(0,1)[16] .{ }^{3}$ From the practical point of view, the mean of log-normal process represents the impact of distancedependent path loss varying over longer periods of time. Measurements also show that $g(\cdot)$ exhibits correlation of an exponentially decaying form [17]:

$$
R_{g}(\tau)=E\{g(t) g(t+\tau)\}=\exp \left(-\frac{v|\tau|}{d_{\rho}}\right),
$$

where $E\{\cdot\}$ is the statistical expectation and $d_{\rho}$ is the decorrelation distance. In [17], $d_{\rho}$ is calculated to be $5.75 \mathrm{~m}$ and $350 \mathrm{~m}$ for urban and suburban environments, respectively, which emphasizes the difference between correlation scales of shadowing and fast-fading under practical scenarios. It is important to state that both (3) and (4) correspond to idealized cases which are consistent with experimental results available in the literature. Note also that there are some other studies in the literature related to shadowing models such as static and dynamic shadowing [18]. Yet, the model defined by both (3) and (4) are adopted due to the following two reasons: (R1) It

\footnotetext{
${ }^{3}$ Since the statistics of the slow-fading process are given in terms of power levels, the constant $1 / 2$ is introduced into (3) for notational convenience and easier analysis implying $\ln \left(s^{2}(t)\right)=\mu(t)+\sigma_{G} g(t)$.
} 
is clear that due to the mathematical tractability of both (3) and (4), the analysis will be simpler. (R2) Furthermore, such a sharp (exponential) decay yields pessimistic results in terms of shadowing correlation, which provide some sort of upper bound for the problem considered. Having said this, as will be shown subsequently, it is important to mention that the proposed method is independent of any sort of shadowing correlation model.

Finally, without loss of generality, it is assumed that receiver displacement within the duration of operation is negligibly small compared to the distance between the unknown signal source and the receiver. Therefore, the impact of $\mu(t)$ can be neglected and $s(t)$ is assumed solely to include the impact of shadowing process.

\section{Proposed Method}

In Section II, it is stated that statistics of shadowing and fast-fading processes evolve in different scales on spatial domain. This implies that shadowing process is not expected to vary within relatively short displacements such as in a couple of wavelengths of the transmission. Bearing this in mind, first consider passing the received signal through a low-pass filter whose (normalized) impulse response is given by:

$$
w(t)=\frac{1}{2 T_{A}}\left(\operatorname{sgn}\left(t+\frac{T_{A}}{2}\right)-\operatorname{sgn}\left(t-\frac{T_{A}}{2}\right)\right)
$$

where $\operatorname{sgn}(\cdot)$ is the signum (or sign) function and $T_{A}$ denotes the effective averaging duration. Now, let one focus on the hypothesis $H_{1}$, since it includes both noise and the unknown signal terms. If $r(t)$ is passed through the low-pass filter under the hypothesis $H_{1}$, then, in the light of both (2) and (5):

$$
\begin{aligned}
z(t)= & \int_{-\infty}^{\infty} w(t-\tau)(x(\tau)+n(\tau)) d \tau \\
= & \int_{t-T_{A} / 2}^{t+T_{A} / 2} w(t-\tau) m(\tau) s(\tau) a(\tau) d \tau \\
& +\int_{N_{F}(t)}^{t+T_{A} / 2} w(t-\tau) n(\tau) d \tau
\end{aligned}
$$

is obtained. By choosing $T_{A}$ to be so short that shadowing does not change within, ${ }^{4}(6)$ can be rewritten as:

$$
\begin{aligned}
z(t) & =s(t) \underbrace{\int_{t-T_{A} / 2}^{t+T_{A} / 2} w(t-\tau) m(\tau) a(\tau) d \tau}_{M_{F}(t)}+N_{F}(t) \\
& =s(t) M_{F}(t)+N_{F}(t) .
\end{aligned}
$$

To reveal the impact of shadowing process, first absolute square of output of the low-pass filter is calculated:

$$
z(t) z^{*}(t)=|z(t)|^{2}=Z(t)=\left|s(t) M_{F}(t)+N_{F}(t)\right|^{2}
$$

which can be rewritten as:

$Z(t)=s^{2}(t)\left|M_{F}(t)\right|^{2}+\left|N_{F}(t)\right|^{2}+2 s(t) \Re\left\{M_{F}(t) N_{F}^{*}(t)\right\}$,

\footnotetext{
${ }^{4} \mathrm{~A}$ brief discussion regarding to what extent $T_{A}$ can be considered to be short is given by Footnote 11 in Section IV for both practical scenarios and general cases.
}

where $(\cdot)^{*}$ and $\Re\{\cdot\}$ denote the complex conjugate and the real part of their input, respectively. Second, natural logarithm operator is applied to (8) which yields after some algebraic manipulations:

$$
\ln (Z(t))=\ln \left(s^{2}(t)\left|M_{F}(t)\right|^{2}\right)+\ln \left(\frac{Z(t)}{\left|s(t) M_{F}(t)\right|^{2}}\right) .
$$

Plugging (3) into (9) by neglecting the impact of distancedependent path loss reads:

$\ln (Z(t))=\sigma_{G} g(t)+\underbrace{\ln \left(\left|M_{F}(t)\right|^{2}\right)+\ln \left(\frac{Z(t)}{\left|s(t) M_{F}(t)\right|^{2}}\right)}_{L(t)}$.

It is clear that autocorrelation of (10) will include the shadowing correlation via $g(t)$. Therefore, first let the unbiased estimate of autocorrelation for any random process $\mathcal{X}(t)$ be defined as: ${ }^{5}$

$$
R_{\mathcal{X}}(\tau)=\lim _{T \rightarrow \infty} \frac{1}{T+\tau} \int_{-T / 2}^{T / 2+\tau} \mathcal{X}(t) \mathcal{X}^{*}(t-\tau) d t
$$

Thus, autocorrelation of (10) in terms of (11) becomes:

$$
R_{\ln (Z)}(\tau)=\sigma_{G}^{2} e^{-v|\tau| / d_{\rho}}+R_{L}(\tau)+R_{g L}(\tau)+R_{L g}(\tau) .
$$

In (12), the impact of correlation characteristics of shadowing process can be seen within the first term at right hand side of the equation. However, (12) by itself is still not very conclusive to make any decision regarding the absence/presence of the unknown signal due to the following reasons: (I) the impact of low-pass filtering operation still exists and affects especially the delays (lags) $\tau$ around zero, (II) the autocorrelation estimates are biased with the mean of $\ln (Z(t)),{ }^{6}$ and (III) in the presence of finite support, autocorrelation estimates will fluctuate and make the decision process difficult. As will be shown in what follows, all these concerns can be alleviated by examining the noise-only process through the steps (6)-(11). In the subsequent parts, (12) will be revisited and discussed further in the light of the results to be obtained for the noiseonly case.

For the noise-only case, consider first the ideal scenario where $T \rightarrow \infty$ and $T_{A}=0$. Note that this scenario corresponds to infinite support with no low-pass filtering and immediately degenerates to $N_{F}(t)=n(t)$. In that case, input to the logarithm operator has a chi-square distribution with two degrees of freedom $\left(\chi^{2}(2)\right)$. However, since input to the logarithm operator is generated by the squaring device, one can take advantage of the equality: $\ln \left(|n(t)|^{2}\right)=2 \ln (|n(t)|)$. This way, input-output relationship for the logarithm operator can be expressed in terms of Rayleigh distribution, which provides analytical tractability in the subsequent steps. Because $\mathcal{Y}(t)=|n(t)|$ is Rayleigh distributed, its probability density function (PDF) is given by:

$$
p_{\mathcal{Y}}(y)= \begin{cases}\frac{y}{\alpha} e^{\left(-\frac{y^{2}}{2 \alpha}\right)}, & 0 \leq y \\ 0, & y<0\end{cases}
$$

${ }^{5}$ Here, it must be stated that the reliability of autocorrelation estimates at larger lags is very low; therefore, the estimates at larger delays should be disregarded.

${ }^{6}$ As will be shown subsequently, mean of $\ln (Z(t))$ actually plays a crucial role in the decision step. Therefore, it is not removed from (12). 
where $\alpha$ is the mode parameter of the distribution satisfying $\mu_{\mathcal{Y}}=\alpha \sqrt{\frac{\pi}{2}}$ with $\mu_{\mathcal{Y}}$ being the mean of the PDF of $\mathcal{Y}(t)$ and $\alpha=\sqrt{\sigma_{N}^{2} / 2}$. Therefore, output of the logarithm, say $\mathfrak{X}(t)$, forms a time series that is composed of log-Rayleigh distributed values:

$$
\mathfrak{X}(t)=\ln (\mathcal{Y}(t))=\ln (|n(t)|),
$$

with the following PDF:

$$
p_{\mathfrak{X}}(\mathfrak{x})=\frac{e^{2 \mathfrak{x}}}{\alpha} \exp \left(-\frac{e^{2 \mathfrak{x}}}{2 \alpha}\right)
$$

for all $\mathfrak{x} \in \mathbb{R}$. Next, let:

$$
r^{\prime}(t)=\lim _{A \rightarrow 0} A \cos \left(2 \pi f_{A} t+\phi_{A}\right)+n(t),
$$

where $A, f_{A}$, and $\phi_{A}$ are some arbitrary amplitude, frequency, and phase values, respectively. Note that (16) is equivalent of hypothesis $H_{0}$ in the limiting sense. If $r^{\prime}(t)$ follows through the steps (6)-(11), then output of the correlator is given by [19]:

$$
\begin{aligned}
\Psi(\tau) & =\sum_{\substack{i=1 \\
(i+l \text { even })}}^{\infty} \varphi_{N}^{i}(\tau) \sum_{l=1}^{i}\left(\begin{array}{c}
(i+l) / 2-1 \\
(i-l) / 2
\end{array}\right) \Upsilon^{m} \\
& \times{ }_{1} F_{1}^{2}((i+l) / 2 ; l+1 ;-\Upsilon) / l ! l(i+l) \\
& +\frac{1}{4} \sum_{i=1}^{\infty}{ }_{1} F_{1}^{2}(i ; 1 ;-\Upsilon) \varphi_{N}^{2 i}(\tau)+\left(\ln (A)+\frac{1}{2} \mathfrak{E}_{1}(\Upsilon)\right)^{2},
\end{aligned}
$$

where $\varphi_{N}(\cdot)$ is the normalized autocorrelation estimates of the quadrature components (i.e., $\left.n_{Q}(\cdot)\right)$ of $n(t), \Upsilon$ is the signalto-noise ratio (SNR) and defined to be $\Upsilon \triangleq A^{2} /\left(2 \sigma_{N}^{2}\right)$, ${ }_{1} F_{1}^{2}(\cdot ; \cdot ; \cdot)$ is the confluent hypergeometric function, and $\mathfrak{E}_{1}(\cdot)$ is the exponential integral [20]. Since the purpose is to obtain the characteristics of the noise-only process, one can consider (17) by expanding $\mathfrak{E}_{1}(\cdot)$ into power series for $A \rightarrow 0$ (or equivalently for $\Upsilon \rightarrow 0$ ). This allows one to see that (17) is dominated by the cross-noise terms as $\Upsilon$ diminishes and can be expressed after some manipulations as:

$$
R_{\mathfrak{X}}(\tau) \triangleq \lim _{\Upsilon \rightarrow 0} \Psi(\tau) \cong \frac{1}{4} \sum_{i=1}^{\infty} \frac{\varphi_{N}^{2 i}(\tau)}{i^{2}}+\Phi\left(\sigma_{N}\right),
$$

where $\Phi\left(\sigma_{N}\right)$ represents a constant that depends on the noise variance $\sigma_{N}^{2}$. Since $\Phi\left(\sigma_{N}\right)$ is a constant, one can readily calculate the variance of $\mathfrak{X}(t)$ by setting $\tau=0$ and ignoring $\Phi\left(\sigma_{N}\right)$ as:

$$
\sigma_{\mathfrak{X}}^{2}=\frac{1}{4} \sum_{i=1}^{\infty} \frac{1}{i^{2}}=\frac{\pi^{2}}{24} .
$$

Recalling that $\mathfrak{X}(t)$ is a non-zero mean process (i.e., $\mu_{\mathfrak{X}} \neq 0$ ) due to the non-linear transformation applied, one can conclude that:

$$
\Phi\left(\sigma_{N}\right)=\mu_{\mathfrak{X}}^{2}
$$

holds in (18), since $R_{\mathfrak{A}}(\tau)=E\left\{\mathfrak{A}(t) \mathfrak{A}^{*}(t+\tau)\right\}$ and $R_{\mathfrak{A}}(0)=\sigma_{\mathfrak{A}}^{2}+\mu_{\mathfrak{A}}^{2}$ for any stationary stochastic process $\mathfrak{A}(t)$ with $\mu_{\mathfrak{A}} \neq 0$.

Then by assuming $\sigma_{N}$ to be unity for the sake of simplicity, $\mu_{\mathfrak{X}}$ can be calculated via (13) and (15)-(18) as:

$$
\mu_{\mathfrak{X}}=\ln (\alpha)+\frac{\ln (2)-\gamma}{2}=\ln \left(\frac{1}{\sqrt{2}}\right)+\frac{\ln (2)-\gamma}{2}=-\frac{\gamma}{2},
$$

where $\gamma$ is Euler-Mascheroni (or sometimes referred to solely as Euler's) constant and given by $\gamma=-\int_{0}^{\infty} \ln (u) e^{-u} d u .^{7}$ In (18), it is clear that at larger delays (lags) $\tau$, the autocorrelation estimates exhibit an asymptotic behavior and converges $\Phi\left(\sigma_{N}\right)$, which is a function of noise variance. However, it is desired that the method proposed is independent of noise variance $\sigma_{N}^{2}$. Thus, normalizing the autocorrelation estimates with the signal power (i.e., with the value at $\tau=0$ ) will yield the following constant: ${ }^{8}$

$$
\Phi^{\prime}=\frac{\Phi\left(\sigma_{N}\right)}{R_{\mathfrak{X}}(0)}=\frac{\mu_{\mathfrak{X}}^{2}}{\mu_{\mathfrak{X}}^{2}+\sigma_{\mathfrak{X}}^{2}} .
$$

Up until this point, $T_{A}$ is assumed to be zero corresponding to omitting the low-pass filtering operation for the noise-only case. It is evident that such a filtering operation prior to the logarithmic detector will not change the convergent behavior of $\Phi^{\prime}$; however, it will solely change the value of $\Phi^{\prime}$ to a new constant, say $\Phi_{T_{A}}^{\prime \prime}$. Due to the space limitations, derivations regarding the value of $\Phi_{T_{A}}^{\prime \prime}$ is omitted and numerical results are presented in Section IV for different $T_{A}$ values.

As final step, one might want to obtain an approximation for (18) in the presence of finite support, since it causes fluctuations around $\Phi_{T_{A}}^{\prime \prime}$ at larger $\tau$ values as stated earlier in (III). Thus, the following unbiased estimator is applied to the autocorrelation estimates in (12) prior to the decision step:

$$
V_{U}=\frac{1}{U} \int_{T_{A}}^{T_{A}+U} R_{\ln (Z)}(\tau) d \tau
$$

where $U$ denotes the effective integration time. It is clear that when $T_{A}=0^{+}$and $U \rightarrow \infty$, then $V_{U} \rightarrow \Phi^{\prime}$.

Based on the discussion related to steps between (12) and (23), it is evident that when $x(t) \neq 0$, shadowing correlation will still influence the autocorrelation estimates at larger delays (lags) $\tau$ and not give rise to a rapid decay. In other words, when an unknown signal is abscent, a sharp decay is expected at the output of the correlator due to the aforementioned secondorder statistical characteristics of noise. However, when an unknown is signal present, although there will still be a decay at the output of the correlator, that decay will never reach the level where noise-only case lies in spite of the exponential decaying assumed for the shadowing in (4)..$^{9}$ Therefore, one can conclude that:

$$
\Phi_{T_{A}}^{\prime \prime}<V_{U}
$$

always holds. Moreover, as shown earlier, $\Phi_{T_{A}}^{\prime \prime}$ implies that no such measurement is required to determine a specific threshold.

At this point, it is worth mentioning the practical implications of the integration limits and of integration duration in (23). In parallel to the earlier dicussions, first and foremost, $T_{A}$ should be kept as low as possible in order not to violate the assumption regarding the invariance of shadowing process in (8). In that sense, it is plausible to think that $T_{A}$ is selected to be the lowest non-zero value possible at the receiver. For instance, considering the discrete sampling operation, $T_{A}$ can be selected to be $k \Delta t$, where $2 \leq k$ with $\Delta t=1 / f_{s}$ denoting

\footnotetext{
${ }^{7}$ First five digit of $\gamma$ in decimal form is $\gamma \cong 0.57721 \ldots$
}

${ }^{8}$ When $\sigma_{N}$ is unity: $\Phi^{\prime}=\frac{\gamma^{2}}{\gamma^{2}+\pi^{2} / 6} \cong 0.16843 \ldots$ for the first five significant digits in decimal.

${ }^{9}$ This is very critical because of the reason (R2) stated in Section II. Considering the fact that the proposed method is independent of any specific correlation model for shadowing, $\Phi_{T_{A}}^{\prime \prime}$ constitutes the lower bound for the problem considered here. 
the sampling interval of the receiver. ${ }^{10}$ This way, it is ensured that (8) still holds. Second, in contrast to $T_{A}, U$ needs to be chosen as large as possible to obtain better estimates $V_{U}$ in (23). In this regard, $U$ is actually bounded by the memory limitations of the receiver while disregarding the values at larger delays (lags) due to the reliability concerns stated in Footnote 5 .

In the light of the aforementioned discussions, finally, the decision step can be given as follows:

$$
\mathcal{D}= \begin{cases}H_{0}, & 0 \leq \mathcal{M}, \\ H_{1}, & \text { otherwise }\end{cases}
$$

where the decision metric is given by $\mathcal{M}=\left(\Phi_{T_{A}}^{\prime \prime}-V_{U}\right)$. The block diagram of the method proposed is given in Figure 1 along with the corresponding steps applied in the analysis.

\section{Numerical Results AND Discussions}

The receiver is assumed to be mobile with an average speed value of $v=10 \mathrm{~m} / \mathrm{s}$ and to operate on $2 \mathrm{GHz}$. In parallel, the fast-fading channel is assumed to have a Rayleigh distributed amplitude with a Doppler spectrum of Jakes' type. Spatially correlated log-normal shadowing is imposed on the signal with $\sigma_{G}=\{4.3,7.5\} \mathrm{dB}$ and $d_{\rho}=\{5.75,350\} \mathrm{m}$ for urban and suburban environments, respectively, as reported in [17]. These two particular environments are selected to investigate the performance of the method proposed, because they exhibit very different characteristics in terms of their decorrelation distances implying different rate of change in their secondorder statistics. The sampling frequency of the receiver is assumed to be fixed at $20 \mathrm{KHz}$ in order to satisfy the condition $f_{D} \ll f_{s}$ as stated in Footnote 10. The impact of path loss is neglected under hypothesis $H_{1}$ by assuming a very significant spatial separation between the unknown source and the receiver with respect to the displacement that the mobile traverses. The effective averaging time of the low-pass integrate-and-dump filter is set to be $0.05 \mathrm{~ms}$ corresponding to the case $k=2$, whereas the total sensing time is set to be $U=50 \mathrm{~ms} .^{11}$

First, one might want to focus on the sampled version of normalized $R_{\ln (|n|)}(\tau)$ with $R_{\ln (|n|)}(0)$, since it constitutes the base of the method proposed and reveals the impact of operations applied between the steps (8)-(24). In Figure 2, two distinct characteristics mentioned earlier can clearly be seen: the impact of filtering and convergence of autocorrelation of the noise passed through logarithmic detector. Note that the impact of filtering reveals itself by forming a gradual decaying from the normalized autocorrelation value at $\tau=0$ to the constant $\Phi^{\prime}$ and $\Phi_{T_{A}}^{\prime \prime}$ s. Here, different $\Phi_{T_{A}}^{\prime \prime}$ values can easily be identified in Figure 2 as saturation points for corresponding $k=\{2,3,4\}$. For instance, $\Phi_{T_{A}}^{\prime \prime}=0.495$ for $k=2$.

Next, the correlation behavior of the unknown signal contaminated by noise can be examined. The results are given in Figure 3 in comparison with the case where unknown signal is absent (noise-only case) for urban environment. In accordance with (24), as the unknown signal dominates, the saturation level for the autocorrelation estimates diverges because shadowing correlation prevails (12) and maintains larger correlation values as expected.

Urban environment settings are applied to both the method proposed and the energy detection methods to obtain a comparative performance investigation. Comparison is established by receiver operating characteristic (ROC), that is probability

\footnotetext{
${ }^{10}$ Of course, this conclusion depends on relatively larger sampling rates $f_{s}$ at the receiver which satisfy $f_{D} \ll f_{S}$.

${ }^{11}$ Note that, $T_{A}$ is suggested to be $85 \mathrm{~ms}$ in [21] as a general-purpose value considering a plausible range of mobile velocities. Therefore, the $T_{A}$ value selected here implies that shadowing process can safely be assumed to be invariant under practical scenarios.
}

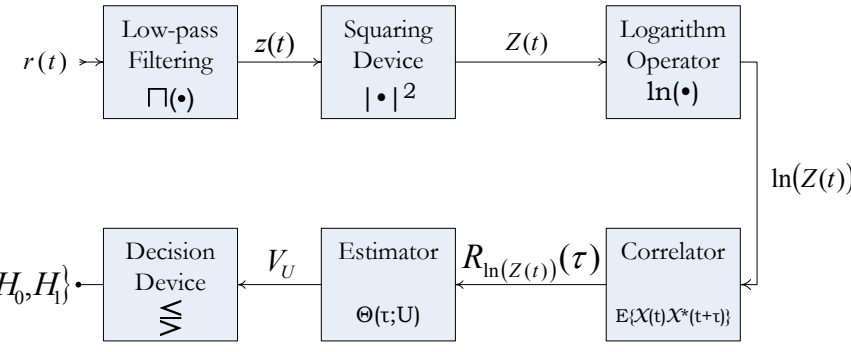

Fig. 1. Block diagram of the method proposed.

of detection $\left(P_{D}\right)$ with respect to probability of false alarm $\left(P_{F}\right)$. In the literature, complementary ROC, which focuses on probability of miss-detection $\left(P_{M D}\right)$ with respect to $P_{F}$, is widely employed as well. In this study, complementary ROC will be considered. The results are plotted in Figure 4 for different SNR values. As seen from Figure 4, the proposed method performs better than the energy detection even for low SNR values.

The impact of decorrelation distance $d_{\rho}$ on the method proposed can be investigated in a better way by looking at output of the correlator. The results are given in Figure 5 for two environment instances under 0dB SNR. Figure 5 indicates that the shorter the $d_{\rho}$, the more rapid decay in (12), since extremely large $d_{\rho}$ values such as $350 \mathrm{~m}$ in suburban environments do not allow (12) to decay rapidly as expected.

\section{CONClusion}

In this study, a spectrum sensing method that is based on the second-order statistical characteristics of the received signal is proposed for mobile radio receivers. The method proposed takes into account the impact of correlated shadowing and fast-fading process and exploits the spatial occurrence scales of these processes. Upon low-pass filtering the received signal at the baseband, a logarithmic detector is applied right after squaring device and a decision is made by examining the autocorrelation characteristics of output.

The proposed method reveals that spatially correlated shadowing is extremely important in spectrum sensing for mobile receivers. It is clear that ignoring shadowing correlation might lead to optimistic results for second-order statistics-based methods and must be avoided.

\section{ACKNOWLEDGMENT}

This work is supported by Qatar National Research Fund $(\mathrm{QNRF})^{12}$ grant through National Priority Research Program with the project number 08-101-2-025.

\section{REFERENCES}

[1] (2005, Aug.15,) Spectrum Occupancy Measurements Location 4 of 6 : Republican National Convention, New York City, New York August 30, 2004-September 3, 2004. Shared Spectrum Company. Vienna, VA, U.S.A.

[2] J. Mitola III, "Cognitive radio an integrated agent architecture for software defined radio," Ph.D. dissertation, KTH Royal Institute of Technology, Stockholm, Sweden, May 8, 2000. [Online]. Available: http://www.it.kth.se/ jmitola/Mitola_Dissertation8_ Integrated.pdf

[3] S. Yarkan and H. Arslan, "Binary Time Series Approach to Spectrum Prediction for Cognitive Radio," in Proc. 66th IEEE Vehicular Technology Conference, [VTC 2007-Fall], Baltimore, Maryland, U. S. A., Sep. 30- Oct. 3, 2007, pp. 1563-1567.

[4] J. G. Proakis, Digital Communications. New York, U. S. A.: McGrawHill International Editions, New York, 2001.

\footnotetext{
${ }^{12} \mathrm{QNRF}$ is an initiative of Qatar Foundation.
} 


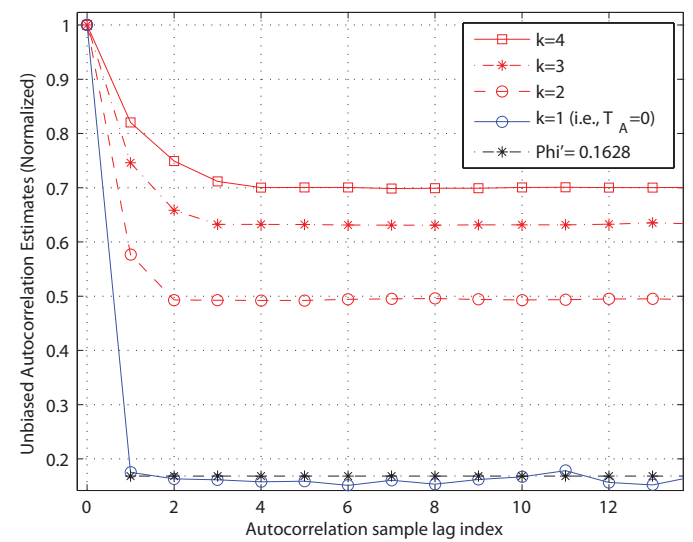

Fig. 2. Correlator output of $H_{0}$ for $k=1, \overline{4}$, as $k=1$ implies $T_{A}=0$ $\left(\Phi^{\prime}\right.$ is labeled as $\mathrm{Phi}^{\prime}$ and $\left.\approx 0.1628\right)$.

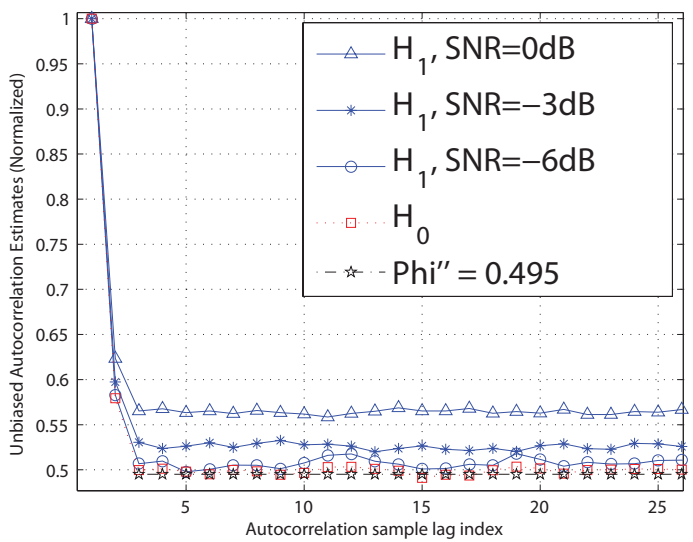

Fig. 3. Correlator output for $x(t) \neq 0$ with $k=2$ (The plot belonging to noise-only case is maintained for comparison purposes).

[5] H. Tang, "Some physical layer issues of wide-band cognitive radio systems," in Proc. First IEEE International Symposium on New Frontiers in Dynamic Spectrum Access Networks (DySPAN), Baltimore, Maryland, U.S.A., Nov. 8-11, 2005, pp. 151-159.

[6] A. Sahai, R. Tandra, S. M. Mishra, and N. Hoven, "Fundamental design tradeoffs in cognitive radio systems," in TAPAS '06: Proceedings of the first international workshop on Technology and policy for accessing spectrum, Boston, Massachusetts, U. S. A., Aug. 5, 2006, pp. 1-9.

[7] M. Öner and F. Jondral, "Cyclostationarity based air interface recognition for software radio systems," in Proc. IEEE Radio and Wireless Conference, Atlanta, Georgia, U.S.A., Sep. 19-22, 2004, pp. 263-266, T4B. 4

[8] A. Fehske, J. Gaeddert, and J. H. Reed, "Some physical layer issues of wide-band cognitive radio systems" in Proc. First IEEE International Symposium on New Frontiers in Dynamic Spectrum Access Networks (DySPAN), Baltimore, Maryland, U.S.A., Nov. 8-11, 2005, pp. 144 150.

[9] T. Ikuma and M. Naraghi-Pour, "Autocorrelation-based spectrum sensing algorithms for cognitive radios," in Proceedings of 17th International Conference on Computer Communications and Networks, (ICCCN '08), St. Thomas, U.S. Virgin Islands, Aug. 3-7, 2008, pp. 1-6.

[10] R. K. Sharma and J. W. Wallace, "Improved spectrum sensing by utilizing signal autocorrelation," in Proc. IEEE 69th Vehicular Technology Conference, (VTC Spring), Barcelona, Spain, Apr. 26-29, 2009, pp. 1-5.

[11] H. Urkowitz, "Energy detection of unknown deterministic signals," Proceedings of the IEEE, vol. 55, no. 4, pp. 523-531, Apr. 1967.

[12] V. I. Kostylev, "Energy detection of a signal with random amplitude," in Proc. IEEE International Conference on Communications (ICC), vol. 3 , New York, New York, U.S.A., Apr. 28- May 2, 2002, pp. 1606-1610.

[13] T. Yücek and H. Arslan, "A survey of spectrum sensing algorithms for cognitive radio applications," IEEE Communications Surveys Tutorials, vol. 11, no. 1, pp. 116-130, first-quarter 2009.

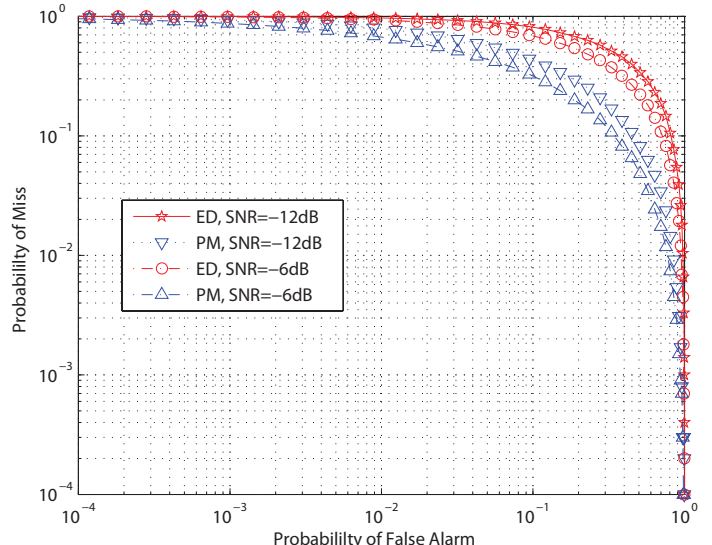

Fig. 4. Complementary ROC curves of both energy detector and the method proposed for different SNR levels in urban environment $(k=2)$.

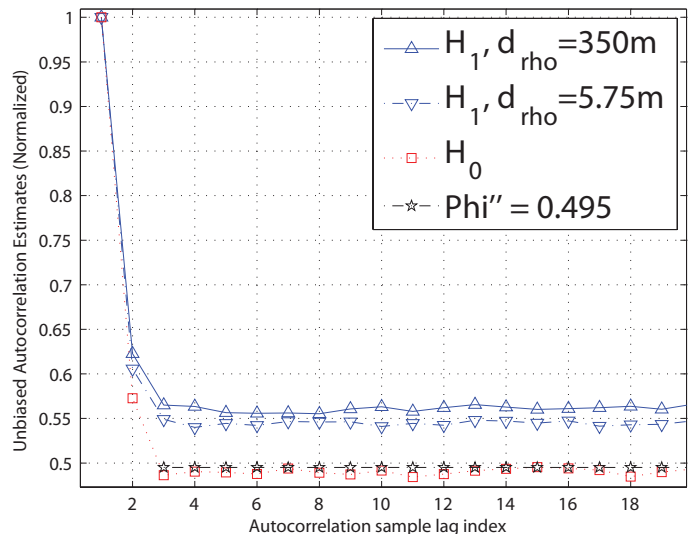

Fig. 5. The impact of different environments having different decorrelation distances on output of the correlator block $(k=2)$.

[14] G. L. Stüber, Principles of Mobile Communications. Kluwer Academic Publishers, 1996, fourth Printing, ISBN: 0-7923-9732-0.

[15] M. Pätzold, U. Killat, and F. Laue, "A Deterministic Digital Simulation Model for Suzuki Processes with Application to a Shadowed Rayleigh Land Mobile Radio Channel" IEEE Transactions on Vehicular Technology, vol. 45, no. 2, pp. 318-331, May 1996.

[16] X. Cai and G. B. Giannakis, "A Two-Dimensional Channel Simulation Model for Shadowing Processes," IEEE Transactions on Vehicular Technology, vol. 52, no. 6, pp. 1558-1567, Nov. 2003.

[17] M. Gudmundson, "Correlation Model for Shadow Fading in Mobile Radio Systems," Electronics Letters, vol. 27, no. 23, pp. 2145-2146, Nov. 7th, 1991

[18] C. Oestges, N. Czink, B. Bandemer, P. Castiglione, F. Kaltenberger , and A. J. Paulraj, "Experimental characterization and modeling of outdoor-to- indoor and indoor-to-indoor distributed channels," IEEE Transactions on Vehicular Technology, 2010, to appear.

[19] J. A. Roberts, "Logarithmic detection with postdetection filtering," IEEE Transactions on Aerospace and Electronic Systems, vol. AES-14, pp. 35-43, Jan. 1978 .

[20] M. Abramowitz and I. A. Stegun, Handbook of mathematical functions: with formulas, graphs, and mathematical tables. Courier Dover Publications, 1965

[21] A. J. Goldsmith, L. J. Greenstein, and G. J. Foschini, "Error Statistics of Real-time Power Measurements in Cellular Channels with Multipath and Shadowing," IEEE Transactions on Vehicular Technology, vol. 43 no. 3.P.I, pp. 439-446, Aug. 1994. 